\title{
BIOLOGIA, ECOLOGIA Y UTILIZACION DEL IRAPAY (Lepidocaryum gracile Martius)
}

\author{
Kember M. Mejía*
}

Francis Kahn

\section{RESUMEN}

Lepidocaryum gracile Martius, "irapay" es una pequeña palmera del sotobosque, su estrategia de crecimiento es remarcable debido a la formación de rizomas estoloníferos que constituyen un modo original y eficiente de propagación vegetativa que convierte a esta palmera en la especie más abundante del estrato inferior de algunos bosques de la amazonía peruana.

Sus hojas palmadas, con cuatros segmentos, son utilizadas en el techado de las viviendas. Se presenta una estimación cuantitativa de este uso.

\section{ABSTRACT}

Lepidocaryum gracile $M$ artius, is a small palm, very abundant in the understory of J enaro Herrera forets. Its growth strategy is remarkable due to the formation of creeping and stoloniferous rhizomes which constitute an effective way of vegetative propagation.

Its palmate, four-segments, leaves are used to make house roofs. A quantitative estimation of this use is presented.

Instituto de Investigaciones de la Amazonía Peruana.

Instituto Francés para el desarrollo en Cooperación-ORSTOM. 


\section{INTRODUCCION}

Lepidocaryum gracile Martius, conocida localmente como "irapay", pertenece a la tribu Lepidocaryeae de la subfamilia Calamoideae, caracterizada por sus frutos cubiertos por escamas imbricadas (U hl \& Dransfield 1987). De los 22 géneros que conforman este grupo, solamente 4 (Mauritia, Mauritiella, lepidocaryum y Raphia) se encuentran en América del Sur. Lepidocaryum cuenta con 9 especies (Uhl \& Dransfield 1989); en el Perú se han reportado 2 especies; sin embargo es necesario una revisión del género para definir los nombres válidos.

L. gracile, es notoria por su abundancia en los bosques de "altura", por su dinámica de crecimiento, en el sotobosque, poco común en las palmeras de la amazonía, por su distribución, modificada por el hidromorfismo del suelo y por su uso tradicional en la confección de los techos de las viviendas. En el presente trabajo se desarrolla diversos aspectos biológicos, ecológicos y etnobotánicos del "irapay".

\section{AREA DE ESTUDIO}

Los estudios se realizaron en los bosques cercanos al caserío de Jenaro Herrera, en el bajo río U cayali, 40 55' 18" S; 730 40' 36" W, aproximadamente a $200 \mathrm{~km}$ al Suroeste de la ciudad de Iquitos. La altitud sobre el nivel del mar es de $125 \mathrm{~m}$, el clima es típicamente húmedo tropical con un promedio anual de precipitación de $2.9 \mathrm{~m}$ y temperatura de $26^{\circ} \mathrm{C}$.

\section{DESCRIPCION BOTANICA}

\section{Lepidocaryum gracile $\mathrm{C}$. Martius}

Sinónimo: Lepidocaryum enneaphyllum Barboza R odríguez Lepidocaryum tessmannii Burret

Palmera pequeña, cespitosa, policárpica, dioica. Estípite inerme, con entrenudos marcados, erecto, 3-3.5 m de altura y 3-4 cm de diámetro. Hojas en número de 10-15; palmadas, erectas o semierectas; vaina frecuentemente cubierta por un 
tomento denso, caduco; pecíolo conspicuo, 1-1.2 m de longitud, más o menos redondeado en sección transversal, acanalado en la base, hástula ausente; lamina flabelada 0 más 0 menos orbicular, dividida en 4 segmentos; segmentos espatulado-acuminados, rígidos, radiados desde la base, todos más o menos iguales con espinas cortas y fuertes a lo largo de las nervaduras y los márgenes en la superficie superior; las hojas jóvenes a menudo rojizas.

Inflorescencia interfoliar, estaminada y pistilada similares; la pistilada erecta en floración, péndula en fructificación, de tamaño variable; profil tubular, brácteas tubulares, pedúnculo de 0.8-1 m de largo, ráquis tan largo como el pedúnculo, 2-18 ramas de hasta $15 \mathrm{~cm}$ de largo, brácteas del ráquis similares a las del pedúnculo. Flores simétricas, blanco amarillentas, fuertemente perfumadas en antésis; flores estaminadas, cáliz tubular 3 lobado, pétalos excediendo el cáliz, concrescentes en la base, estambres 6 , pistilodio menudo; flores pistiladas de igual tamaño que las pistiladas, cáliz tubular, 3 lobado, corola excediendo el cáliz, tubular en casi 1/3 de su longitud, tres lóbulos valvados, 6 estaminodios adnatos a la base de los segmentos, ovario trilocular, triovulado, más o menos redondeado, cubierto con filas verticales de escamas, estilo cónico. Fruto oblongo, en ocasiones globoso, de hasta $3 \mathrm{~cm}$ de largo, $1.5-2 \mathrm{~cm}$ de diámetro, cubierto con escamas imbricadas, dispuestas en series verticales, rojo-anaranjado intenso en la madurez, estigma apical remanente; generalmente 1 semilla, endosperma homogéneo, embrión lateral. Su área de distribución comprende el Oeste y centro de la cuenca amazónica.

\section{DINAMICA DE CRECIMIENTO}

Las agregaciones de L. gracile, son formadas por ramas basales que corresponde a los patrones de crecimiento definido como modelo de Tomlinson "Esta arquitectura resulta del desarrollo repetido de módulos ortotrópicos equivalentes en la forma de ramas basales que son, inicialmente, restringidos a las regiones epicotiledonares del eje de las plántulas (el primer módulo) y los nodos basales en los ejes subsecuentes" (Hallé, 1968; Hallé \& Oldeman, 1970; Hallé, Oldeman \& Tomlinson, 1978).

Numerosas palmeras amazónicas desarrollan este modelo de crecimiento; la originalidad del "irapay" se manifiesta en la producción de rizomas estoloníferos rastreros, forma escamas foliares y emite raíces a todo lo largo de la parte inferior del rizoma. Cada eje produce varios rizomas desde la base, de 0.5 a 1 $\mathrm{cm}$ de diámetro y hasta $2 \mathrm{~m}$ de longitud, que se extiende a $5 \mathrm{~cm}$ bajo 0 sobre la 
superficie del suelo. Durante la fase de expansión horizontal el ápice crece verticalmente para formar un nuevo tallo, de mayor diámetro que el rizoma.

L as palmeras amazónicas raramente forman rizomas estoloníferos; solamente los encontramos en Iriartella setigera (M art) Wendl. (Kahn 1986) y Lepidocaryum gracile $M$ artius. En las palmeras del paleotrópico es más extendido; así se há descrito para la especie africana Podococcus barteri Mann. et Wendl. (Bullock 1980) y para los géneros A siáticos Salacca (Corner 1966) y A renga. Calamus y otras palmeras lianescentes (ratanes) desarrollan diversos tipos de rizomas descritos por Dransfield (1978).

\section{ABUNDANCIA DEL IRAPAY}

La densidad del "irapay" fué inventariada en dos parcelas de $0.2 \mathrm{~h}$ cada una. U no de ellos ubicado en un típico bosque de altura ${ }^{1}$, sobre suelo ferralítico, bien drenado. En este tipo de bosque el dosel superior, $40 \mathrm{~m}$ de altura, es dominado por diversas especies de Lecythidaceae, Sapotaceae y Caesalpinaceae, con un diámetro promedio de 1.5 m, DBH. (Marmillod 1982). La otra parcela, ubicada en un bosque de quebrada, sobre suelo de podsol hidromórfico, estacionalmente inundado y pobremente drenado. En éstos bosques el dosel superior es dominado por Mauritia flexuosa, L. f., Oenocarpus bataua Mart. y Euterpe precatoria $M$ art., con una baja densidad de dicotiledóneas arbóreas. A mbos bosques cubren bastas áreas en la región de estudio.

En cada una de estas parcelas hemos contado todos los ejes de Lepidocaryum gracile $\mathrm{M}$ artius con altura igual o mayor a un metro.

En el bosque de altura fueron inventariados 508 ejes, (2,540/ha); M armillod (1982), en un bosque del mismo tipo, calculó 3,500 ejes por ha, a partir de un inventario sobre 0.7 ha. La variación de la densidad de un lugar a otro está posiblemente relacionado a la dinámica del bosque.

En el bosque de quebrada, el número de ejes contados fué apenas de 266 (1,330/ha). El "irapay" es menos abundante sobre suelos hidromórficos, sin embargo está bien representado, lo que demuestra su capacidad de tolerar condiciones anaeróbicas en suelos inundados estacional mente. Granville (1978) y Sist (1985) describen un comportamiento análogo en Astrocaryum paramaca

1 El término "bosque de altura" corresponde al concepto brasileño de "bosque de tierra firme". Este último es más conocido y designa bosques de latifoliadas sobre suelos lateríticos amarillos o rojos de buen drenaje. 
$M$ art. una especie del sotobosque de la Guyana Francesa; las poblaciones de esta palmera son menos densas sobre suelos hidromórficos que sobre suelos bien drenados.

Sin embargo éste comportamiento no es común a todas las especies de palmeras. U na clara distribución de las palmeras, con relación al drenaje del suelo ha sido descrita por Kahn y Castro (1985) en la amazonía central, cerca de M anaos.

Solamente dos especies escapan a esta regla, Iriartea exorhiza $M$ art. e Iriatella setigera ( $M$ art.) Wendl., que habitan tanto sobre suelos hidromórficos como sobre suelos bien drenados de tierra firme; la primera especie se encuentra con mayor frecuencia en los claros naturales recientes, lo que demuestra su temperamento de planta heliófila.

\section{PROPAGACION VEGETATIVA}

Todos los ejes de "irapay" fueron excavados en una parcela de $50 \mathrm{~m} 2$ en el bosque de tierra firme. Solamente una plántula provenía netamente de germinación; la mayoría de los ejes estaban ligados por rizomas a otros ejes vivos o muertos. En la base de los ejes aislados persistía un rezago del rizoma inicial; lo que indica que la multiplicación vegetativa es bien desarrollada en la especie. A unque se ha observado que el período de fructificación del "irapay" se extiende de marzo hasta mayo, en la estación de mayor precipitación y de setiembre a octubre, durante la estación de menor precipitación (M ejía, inéd.). La producción de rizomas a partir de un eje originado por germinación parece ser precóz, lo cual hace difícil la evaluación de la importancia respectiva de los dos modos de reproducción, sexual y vegetativa. El modo de crecimiento de esta especie, por ramificación basal y formación de rizomas repentes, constituye un proceso de multiplicación vegetativa que le permite invadir el sotobosque de trecho en trecho.

En la mayoría de las especies que responden al modelo de Tomlinson, la ramificación basal llega a constituir una mata que contiene, en algunos casos, más de veinte ejes, como por ejemplo en Euterpe oleracea $M$ art., pero estas matas quedan aisladas. En éste caso, el proceso de ramificación contribuye más al mantenimiento de la población asegurando la producción de ejes fructíferos por un período largo que a la ocupación del espacio (Hallé et al,1978). Aún en Iriartella setigera ( $M$ art.) Wendl. que produce de 4 a 8 rizomas repentes, éstos originan solamente 1 ó 2, raramente 3, nuevos ejes erectos cuando, por 10 general, la palmera madre ha muerto. En las formaciones vegetales estudiadas, L epidocaryum gracile, es la especie más abundante en el estrato inferior hasta los $6 \mathrm{~m}$, debido a su propagación vegetativa por rizomas estoloníferos. 


\section{UTILIZACION DE IRAPAY}

Las hojas del "irapay" son utilizadas tradicionalmente en el techado de las viviendas, uso muy difundido en diversos lugares de la llanura amazónica, aún en los barrios marginales de la ciudad de Iquitos (M ejía, 1983, 1988, 1992).

L a cosecha se realiza cortando el peciolo de la hoja aproximadamente a la mitad de su longitud, las que son preparadas en manojos de 100 unidades, que pueden ser fácilmente transportados.

La unidad de cobertura, de los techos, es la "crizneja", la que esta compuesta de un eje o "ripilla", hecha de otra palmera del sotobosque, especialmente, W ettinia augusta Poeppig et Endlicher. Sobre éstos ejes son atados los peciolos de las hojas del "irapay", los cuatro foliolos de cada hojas son entretejidos con dos foliolos de la hoja precedente.

\section{Foto № 1. Tejido de hojas de Irapay}

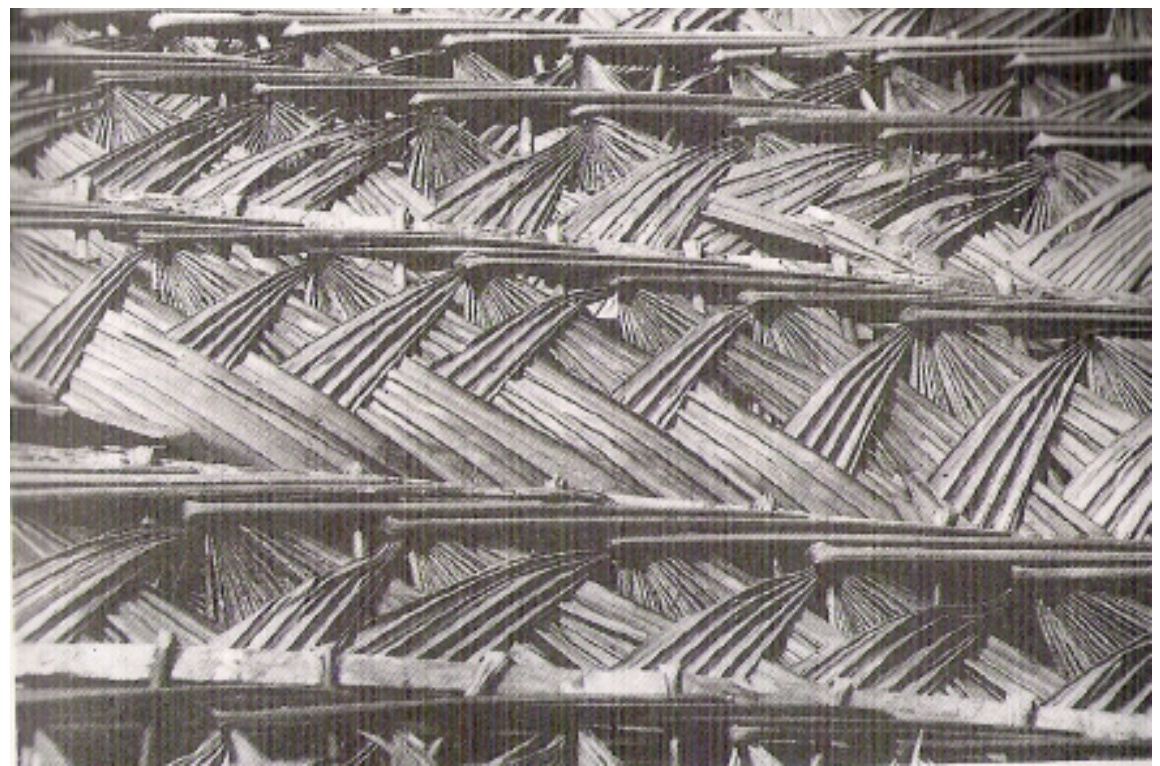


La duración de un techado de hojas de "irapay" varia de cuatro a ocho años dependiendo de la densidad de la unidad de cobertura, el espacio entre ellas y el ángulo de caída del techo.

En J enaro Herrera hemos observado que un techo de $60^{\circ}$ de caída, cubierto con unidades de 150 hojas y distanciamiento de $10 \mathrm{~cm}$ entre "criznejas", el tiempo de duración generalmente es de 8 años.

Para cubrir una vivienda tradicional de $35 \mathrm{~m} 2$, con un techo de dos aguas, se requieren de 160 unidades, 70 por cada lado y 10 por cada frente. Considerando un promedio de 130 hojas por cada crisneja, se necesitarían 20,800 hojas para cubrir una casa de éstas dimensiones. Se pueden colectar un promedio de 4 hojas útiles por cada eje de "irapay", para conseguir las crisnejas requeridas, será, entonces, necesario cosechar las hojas de 5,200 ejes, aproximadamente dos hectáreas en bosques de al ta densidad (2,500 a 3,500 plantas/ha).

En el área rural las "crizneja", se tejen sobre ejes de aproximadamente $3 \mathrm{~m}$ de longitud y $3-4 \mathrm{~cm}$ de ancho; el número de hojas por cada unidad es de 90 a 150. U n ciento de criznejas de estas características cuesta aproximadamente US\$ 26 en la zona rural, mientras que en la ciudad de Iquitos puede alcanzar el precio de US\$ 45 .

L as "criznejas" que se comercializan en la ciudad de Iquitos, tienen una longitud de 2.20 a 2.50 m y constan de 30 a 50 hojas solamente y su precio es de US\$20 el ciento. 


\section{Foto № 2: Transporte de criznejas en la ciudad de Iquitos}

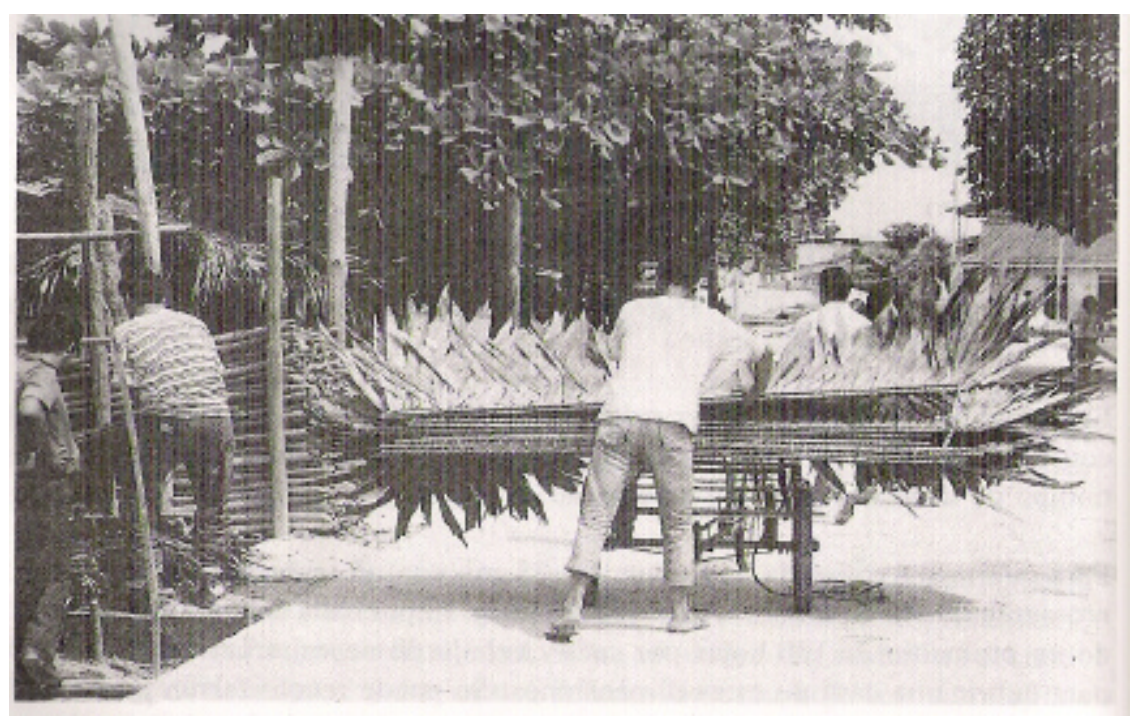

\section{BIBLIOGRAFIA}

BELL, A. D. y P. R. TOMLINSON. 1980. A daptative architecture in rhizomatous plante Bol. Linn. Soc 80(2): pp. 125-169.

BRAKO L., J., L. ZARUCCHI 1993. Catálogo de las angiospermas y gimnospermas del Perú. Monographs in Systematic Botany from the M issouri Botanical Garden, Vol 45.

BULLOCK, S. H. 1980. Demography of an undergromth Palm in littoral. Cameroon. Biotropica 12(4): 247/255.

CORNER E.J.H. 1966 The natural history of palms. Weidenfeld and Nicolson, London.

DRAN SF IELD J. 1978. Growth forms of rain forest palms. In: Tomlinson P.B., Zimmermann M.H. (eds) Tropical trees as living systems. Cambridge U niv. Press, Cambridge, pp. 247-268. 
GRANVILLE, J.J. 1978. Recheeches sur la Flore et la Vegétation Guyanaises. These de doctorat d'état. U niv. de M ontpellier. 272 pp.

HALLE F., R.R.A. OLDEMAN AND P.B. TOMLINSON. 1978 Tropical trees and forests. An architectural analysis. Springer-Verlag. Berlin, Heidelberg, $\mathrm{N}$ ew Y ork.

KAHN F. 1986 Adaptation en forêt tropicale humide: le cas des palmiers amazoniens. Naturalia M onspeliensia HS I'A rbre: pp. 171-189.

----- 1987 The distribution of palms as a function of local topography in A mazonian terra firme forests. Experientia 43: pp. 251-259.

-.--- \& A. CASTRO 1985. The palm community in a forest of central A mazonia, Brazil. Biotropica 17: pp. 210-216.

---- \& K. MEJIA 1987. Notes on the biology, ecology and use of an small A mazonia palm: Lepidocaryum tessmannii. Principes 31: pp.14-19.

---- \& K. MEJIA 1991. The palm communities of two terra firme forests in Peruvian A mazonia. Principes 35: pp. 22-26.

K. MEJIA \& A. CASTRO. 1988 Species richnes and diversity of palms in terra firme forests of A mazonia. Biotropica 20: pp. 266-269.

MARM ILLOD D. 1982 Methodik und ergebnisse von untersuchungen über Zusammensetzung und Aufbau eines Terrasssenwaldes im peruanischen A mazonien. Thesis, Gerog-A ugust U niv., Göttingen.

MEJIA K. 1983. Palmeras y el selvícola amazónico; UNMSM: Museo de Historia Natural, Lima pp. 1-8.

----- 1988. Utilization of palms in eleven mestizo villages of the Peruvian A mazon (U cayali river). Adv. Econ. Bot. 6: pp.130-136.

---- 1992. Las palmeras en los mercados de Iquitos. Bull. Inst. fr. études andines 21(2): pp. 755-769.

SIST P. 1985. Regeneration et dynamique des populations de quelques espèces de palmiers en forêt guyanaise. D.E.A. U niv. Paris VI. 60p. 
UHL N.W., J. DRANSFIELD 1987. Genera palmarum. Allen Press, L aw rence, Kansas, USA. 